Article

\title{
Extended Rectangular $b$-Metric Spaces and Some Fixed Point Theorems for Contractive Mappings
}

\author{
Zead Mustafa ${ }^{1}$, Vahid Parvaneh ${ }^{2}$, Mohammed M.M. Jaradat ${ }^{1, *}$ and Zoran Kadelburg ${ }^{3}$ (i) \\ 1 Department of Mathematics, Statistics and Physics, Qatar University, Doha, P.O. Box 2713, Qatar; \\ zead@qu.edu.qa \\ 2 Department of Mathematics, Gilan-E-Gharb Branch, Islamic Azad University, Gilan-E-Gharb, P.O. Box 0834, \\ Iran; vahid.parvaneh@kiau.ac.ir \\ 3 Faculty of Mathematics, University of Belgrade, Beograd, P.O. Box 11000, Serbia; kadelbur@matf.bg.ac.rs \\ * Correspondence: mmjst4@qu.edu.qa
}

Received: 31 March 2019; Accepted: 19 April 2019; Published: 24 April 2019

check for updates

\begin{abstract}
In this paper, we introduce the class of extended rectangular $b$-metric spaces as a generalization of both rectangular metric and rectangular $b$-metric spaces. In addition, some fixed point results connected with certain contractions are obtained and examples are given to illustrate these results.
\end{abstract}

Keywords: fixed point; generalized metric space; rectangular metric space; $b$-metric space

MSC: Primary 47H10; Secondary 54H25

\section{Introduction}

Fixed points theory has become an important field in mathematics due to its variety of applications in science, economics and game theory. Brouwer's fixed-point theorem states that any continuous mapping on a compact convex set to itself has a fixed point. In addition to their importance in differential and integral equations, Brouwer's theorem and its extension Kakutani theorem for set valued function play a very important role in proving the existence of general equilibrium in market economics and the existence of Nash equilibria in game theory-for more details, see [1,2].

In 2000, Branciari [3] introduced the concept of generalized metric space (rectangular space) as a generalization of normal metric space. In 2015, George et al. [4] introduced the notion of rectangular $b$-metric space as a generalization of rectangular metric space and they presented some fixed point results for contractive mappings.

In this paper, we introduce the notion of extended rectangular $b$-metric spaces which is a combination of properties of rectangular metric spaces and extended $b$-metric spaces. In addition, we obtain some fixed point results dealing with JS-type contraction mappings. Furthermore, we present examples to support these results.

\section{Preliminaries and Known Results}

In 1993, Czerwik [5] introduced the concept of $b$-metric space as follows:

Definition 1 ([5]). Let $X$ be a nonempty set, $s \geq 1$ be a given real number and let $d: X \times X \rightarrow[0,+\infty)$ be a mapping such that for all $a, b, c \in X$ the following conditions hold:

$\left(b_{1}\right) d(a, b)=0$ iff $a=b$;

$\left(b_{2}\right) d(a, b)=d(b, a)$;

$\left(b_{3}\right) d(a, b) \leq s[d(a, c)+d(c, b)]$. 
Then, $(X, d)$ is called a $b$-metric space.

For some fixed points results in $b$-metric space and its properties, we refer the reader to [5-18]. Recently, Parvaneh [19] introduced the concept of extended $b$-metric spaces as follows.

Definition 2 ([19]). Let $X$ be a nonempty set. A function $d: X \times X \rightarrow R^{+}$is a p-metric if there exists a strictly increasing continuous function $\Omega:[0, \infty) \rightarrow[0, \infty)$ with $t \leq \Omega(t)$ such that for all $a, b, c \in X$, the following conditions hold:

$\left(p_{1}\right) d(a, b)=0$ iff $a=b$,

$\left(p_{2}\right) d(a, b)=d(b, a)$,

$\left(p_{3}\right) d(a, c) \leq \Omega(d(a, b)+d(b, c))$.

Then, the pair $(X, d)$ is called a p-metric space or an extended b-metric space.

A $b$-metric is a $p$-metric, when $\Omega(t)=s t, s \geq 1$ while a metric is a $p$-metric, when $\Omega(t)=t$.

Proposition 1 ([19]). Let $(X, d)$ be a metric space and let $\rho(a, b)=\xi(d(a, b))$, where $\xi:[0, \infty) \rightarrow[0, \infty)$ is a strictly increasing continuous function with $t \leq \xi(t)$ and $0=\xi(0)$. Then, $\rho$ is a p-metric with $\Omega(t)=\xi(t)$.

The following example is constructed using the above proposition.

Example 1. Let $(X, d)$ be a metric space and let $\rho(a, b)=e^{d(a, b)} \sec ^{-1}\left(e^{d(a, b)}\right)$. Then, $\rho$ is a p-metric with $\Omega(t)=e^{t} \sec ^{-1}\left(e^{t}\right)$.

In 2000, Branciari [3] introduced the concept of generalized metric space (rectangular space) as follows:

Definition 3 ([3]). Let $X$ be a nonempty set and let $d: X \times X \rightarrow[0,+\infty)$ be a mapping such that for all $a, b \in X$ and all distinct points $c, d \in X$, each distinct from $a$ and $b$, the following satisfied:

$\left(r_{1}\right) d(a, b)=0$ iff $a=b$;

$\left(r_{2}\right) d(a, b)=d(b, a)$;

$\left(r_{3}\right) d(a, b) \leq d(a, c)+d(c, d)+d(d, b)$ (rectangular inequality).

Then, $(X, d)$ is called a generalized metric space (g.m.s.) or rectangular space.

This metric attracted many authors and they obtain many results in this setting (see the references cited in [20-22]). One can notice that each metric space is a generalized metric space, but the converse need not be true (see $[23,24])$. Several authors show that the topology of g.m.s. need not be Hausdorff-see examples in [25-27]. The topological structure of g.m.s. is not compatible with the topology of ordinary metric space (see [24] (Example 7)), so it is not easy to deal with g.m.s. Therefore, this concept is very interesting for researchers.

Definition 4 ([4]). Let $X$ be a nonempty set, $s \geq 1$ be a given real number and let $d: X \times X \rightarrow[0,+\infty)$ be a mapping such that for all $a, b \in X$ and all distinct points $c, d \in X$, each distinct from $a$ and $b$, the following satisfied:

$\left(b_{r 1}\right) d(a, b)=0$ iff $a=b$;

$\left(b_{r 2}\right) d(a, b)=d(b, a)$;

$\left(b_{r 3}\right) d(a, b) \leq s[d(a, c)+d(c, d)+d(d, b)]$ (b-rectangular inequality).

Then, $(X, d)$ is called a rectangular b-metric space.

The following are some easy examples of rectangular $b$-metric spaces. 
Example 2 ([25]). Let $(X, \rho)$ be a g.m.s. and $p \geq 1$ be a given real number. Let $d(x, y)=(\rho(x, y))^{p}$. Then, $(X, d)$ is a rectangular $b$-metric space with $s \leq 3^{p-1}$.

Example 1.1 in [27] clarifies that some properties of metric spaces need not be true in g.m.s. (and so in rectangular $b$-metric space).

Motivated by [28], Hussain et al. [29] used $\Psi$ to stand for the set of all functions $\psi:(0, \infty) \rightarrow(1, \infty)$ satisfying the following conditions:

(1) $\psi$ is non-decreasing;

(2) for each sequence $\left\{t_{n}\right\} \subseteq(0, \infty), \lim _{n \rightarrow \infty} \psi\left(t_{n}\right)=1$ if and only if $\lim _{n \rightarrow \infty} t_{n}=0$;

(3) there exist $r \in(0,1)$ and $\ell \in(0, \infty]$ such that $\lim _{t \rightarrow 0^{+}} \frac{\psi(t)-1}{t^{r}}=\ell$.

(4) $\psi(a+b) \leq \psi(a) \psi(b)$ for all $a, b>0$.

Then, they introduced new concepts of generalized contractive mappings and obtained sufficient conditions for the existence of fixed points for mappings from these classes on complete metric spaces and complete $b$-metric spaces. In particular, they claimed that their results extend theorems of Ćirić, Chatterjea, Kannan and Reich. On the other hand, Jiang et al., by removing the condition (3), proved that in a metric space $(X, d), D(x, y)=\ln (\psi(d(x, y)))$ defines a metric on $X$ (Lemma 1 of [30]). They, also proved that the results in [29] are not real generalizations of Ćirić contractive principle.

From now on, we denote by $\Theta$ the set of all functions $\psi:[0, \infty) \rightarrow[1, \infty)$ satisfying the following conditions:

$\left(\psi_{1}\right) \psi$ is a continuous strictly increasing function;

$\left(\psi_{2}\right)$ for each sequence $\left\{t_{n}\right\} \subseteq(0, \infty), \lim _{n \rightarrow \infty} \psi\left(t_{n}\right)=1$ if and only if $\lim _{n \rightarrow \infty} t_{n}=0$.

Note that, in general, the conditions of $\Theta$ do not guarantee that the metric $d$ generates a new metric $D(x, y)=\ln (\psi(d(x, y)))$, due to the absence of condition $\left(\psi_{4}\right)$.

Remark 1 ([29]). The following are some examples of functions in $\Theta$ :

$$
\begin{aligned}
& \psi(t)=e^{t} \\
& \psi(t)=\cosh t \\
& \psi(t)=1+\ln (1+t) \\
& \psi(t)=e^{t e^{t}} \\
& \psi(t)=\frac{2+2 \ln (1+t)}{2+\ln (1+t)} \\
& \psi(t)=\frac{2 \cosh t}{1+\cosh t} \\
& \psi(t)=e^{\sqrt{t e^{t}}} \\
& \psi(t)=e^{\sqrt{t e} \sqrt{t}}
\end{aligned}
$$

Note that $\psi(t)=e^{t e^{t}}$ does not belong to $\Psi$, since condition (4) does not satisfy.

We recall the following:

Theorem 1 ([28], Corollary 2.1). Let $(X, d)$ be a complete metric space and let $T: X \rightarrow X$ be a given map. Suppose that there exist $\psi \in \Psi$ and $k \in(0,1)$ such that

$$
x, y \in X, \quad d(T x, T y) \neq 0 \Rightarrow \psi(d(T x, T y)) \leq \psi(d(x, y))^{k} .
$$

Then, T has a unique fixed point.

Observe that the Banach contraction principle follows immediately from the above theorem. 


\section{New Definition and Basic Properties}

We start this section by introducing the definition of Extended rectangular $b$-metric space.

Definition 5. Let $A$ be a nonempty set, $\Omega:[0, \infty) \rightarrow[0, \infty)$ be a strictly increasing continuous function with $t \leq \Omega(t)$ for all $t>0$ and $0=\Omega(0)$ and let $\widetilde{r}: A \times A \rightarrow[0,+\infty)$ be a mapping such that for all $a, b \in A$ and all distinct points $u, v \in A$, each distinct from $a$ and $b$ satisfies the following conditions:

$\left(b_{r 1}\right) \widetilde{r}(a, b)=0$ iff $a=b$

$\left(b_{r 2}\right) \widetilde{r}(a, b)=\widetilde{r}(b, a)$;

$\left(b_{r 3}\right) \widetilde{r}(a, b) \leq \Omega[\widetilde{r}(a, u)+\widetilde{r}(u, v)+\widetilde{r}(v, b)](\Omega$-rectangular inequality).

Then, $(A, \widetilde{r})$ is called an extended rectangular b-metric space (ERbMS).

Note that $\Omega^{-1}(t) \leq t$ for all $t>0$ and $\Omega^{-1}(0)=0$. Obviously, each rectangular $b$-metric space is an ERbMS with $\Omega(t)=s t, s \geq 1$. The following gives some more examples of extended rectangular $b$-metric spaces.

Example 3. Let $\left(A, r_{b}\right)$ be a rectangular $b$-metric space with coefficient $s \geq 1$ and let $\xi:[0, \infty) \rightarrow[0, \infty)$ be a strictly increasing continuous function with $t \leq \xi(t)$ and $\xi(0)=0$. Let $\rho(a, b)=\xi\left(r_{b}(a, b)\right)$. Evidently, for all $a, b \in A$ and for any two distinct points $u, v \in A$, each of which distinct from $a$ and $b$, we obtain

$$
\begin{aligned}
\rho(a, b) & =\xi\left(r_{b}(a, b)\right) \leq \xi\left(s r_{b}(a, u)+s r_{b}(u, v)+s r_{b}(v, b)\right) \\
& \leq \xi\left(s \xi\left(r_{b}(a, u)\right)+s \xi\left(r_{b}(u, v)\right)+s \xi\left(r_{b}(v, b)\right)\right. \\
& =\Omega(s \rho(a, u)+s \rho(u, v)+s \rho(v, b)) .
\end{aligned}
$$

Thus, $(A, \rho)$ is an ERbMS with $\Omega(t)=\xi(s t)$.

The convergence of sequences in ERbMS is introduced in a standard way. The following lemma will be needed in forthcoming results.

Lemma 1. Let $(A, \widetilde{r})$ be an $E R b M S$ with the function $\Omega$. Then, we have the following:

(i) Suppose that $\left\{a_{n}\right\}$ and $\left\{b_{n}\right\}$ are two sequences in $A$ such that $a_{n} \rightarrow a, b_{n} \rightarrow b$ and the elements of $\left\{a, b, a_{n}, b_{n}: n \in \mathbb{N}\right\}$ are totally distinct. Then, we have

$$
\Omega^{-1}(\widetilde{r}(a, b)) \leq \liminf _{n \rightarrow \infty} \widetilde{r}\left(a_{n}, b_{n}\right) \leq \limsup _{n \rightarrow \infty} \widetilde{r}\left(a_{n}, b_{n}\right) \leq \Omega(\widetilde{r}(a, b))
$$

(ii) Let $\left\{a_{n}\right\}$ be a Cauchy sequence in A converging to a. If $a_{n}$ has infinitely many distinct terms, then

$$
\Omega^{-1}(\widetilde{r}(a, b)) \leq \liminf _{n \rightarrow \infty} \widetilde{r}\left(a_{n}, b\right) \leq \limsup _{n \rightarrow \infty} \widetilde{r}\left(a_{n}, b\right) \leq \Omega(\widetilde{r}(a, b))
$$

for all $b \in A$ with $a \neq b$.

Proof. (i) Using the $\Omega$-rectangular inequality, we get that

$$
\widetilde{r}(a, b) \leq \Omega\left[\widetilde{r}\left(a, a_{n}\right)+\widetilde{r}\left(a_{n}, b_{n}\right)+\widetilde{r}\left(b_{n}, b\right)\right]
$$

and

$$
\widetilde{r}\left(a_{n}, b_{n}\right) \leq \Omega\left[\widetilde{r}\left(a_{n}, a\right)+\widetilde{r}(a, b)+\widetilde{r}\left(b, b_{n}\right)\right] .
$$

Taking the lower limit as $n \rightarrow \infty$ in the first inequality and the upper limit as $n \rightarrow \infty$ in the second inequality, we obtain the desired result: 
(ii) If $b \in A$, then for infinitely many $m, n \in \mathbb{N}$,

$$
\widetilde{r}(a, b) \leq \Omega\left[\widetilde{r}\left(a, a_{n}\right)+\widetilde{r}\left(a_{n}, a_{m}\right)+\widetilde{r}\left(a_{m}, b\right)\right]
$$

and

$$
\widetilde{r}\left(a_{n}, b\right) \leq \Omega\left[\widetilde{r}\left(a_{n}, a_{m}\right)+\widetilde{r}\left(a_{m}, a\right)+\widetilde{r}(a, b)\right] .
$$

Taking the lower limit as $n \rightarrow \infty$ in the first inequality and the upper limit as $n \rightarrow \infty$ in the second inequality, we obtain the desired result.

As shown in Example 1.1 of [27], a sequence in an ERbMS may have more than one limits. However, under some constraints, the sequence has a unique limit if it exists. The following lemma is a variant of [28] (Lemma 1.10) and [20] (Lemma 1) and will be used in the main results.

Lemma 2. Let $(A, \widetilde{r})$ be an ERbMS and let $\left\{a_{n}\right\}$ be a Cauchy sequence in $A$ such that $a_{m} \neq a_{n}$ whenever $m \neq n$. Then, $\left\{a_{n}\right\}$ can converge to at most one point.

Proof. Suppose that $\left(a_{n}\right)$ converges to two different points, say $a, b$. Since $a_{m}$ and $a_{n}$ are distinct elements, as well as $a$ and $b$, it is clear that there exists $\ell \in \mathbb{N}$ such that $a$ and $b$ are different from $a_{n}$ for all $n>\ell$. For $m, n>\ell$, the rectangular inequality implies that

$$
\widetilde{r}(a, b) \leq \Omega\left[\widetilde{r}\left(a, a_{m}\right)+\widetilde{r}\left(a_{m}, a_{n}\right)+\widetilde{r}\left(a_{n}, b\right)\right] .
$$

Taking the limit as $m, n \rightarrow \infty$, it follows that $\widetilde{r}(a, b)=0$, i.e., $a=b$. A contradiction.

In this paper, by an ordered ERbMS, understand a triple $(A, \preceq, \widetilde{r})$, where $(A, \preceq)$ is a partially ordered set and $(A, \widetilde{r})$ is an ERbMS.

\section{Main Results}

The following Lemma is needed in a sequel.

Lemma 3. For each $\psi \in \Theta$, we have $\psi(0)=1$.

Proof. Suppose that $\psi(0)=\alpha>1$ and let $t_{n}$ be a sequence in $(0, \infty)$ such that $\lim _{n \rightarrow \infty} t_{n}=0$. Since $\psi$ is a strictly increasing function, we have $\psi\left(t_{n}\right)>\psi(0)=\alpha>1$. Thus, $\lim _{n \rightarrow \infty} \psi\left(t_{n}\right) \geq \alpha>1$ which contradicts the property $\left(\psi_{2}\right)$. Therefore, $\psi(0)=1$.

Let $(A, \widetilde{r})$ be an ERbMS with nontrivial function $\Omega$ (i.e., $\Omega(t) \neq t$ ). Throughout this paper, $\mathcal{F}_{\Omega}$ denotes the class of all functions $\beta:[0, \infty) \rightarrow\left[0, \Omega^{-1}(1)\right)$.

Theorem 2. Let $(A, \preceq, \widetilde{r})$ be a complete ordered ERbMS with nontrivial function $\Omega$. Let $f: A \rightarrow A$ be an increasing mapping with respect to $\preceq$ such that there exists an element $a_{0} \in A$ with $a_{0} \preceq f a_{0}$. Suppose that

$$
\psi\left(\Omega^{2}[\widetilde{r}(f a, f b)]\right) \leq \psi(M(a, b))^{\beta(\widetilde{r}(a, b))}
$$

for some $\beta \in \mathcal{F}_{\Omega}, \psi \in \Theta$ and all comparable elements $a, b \in A$, where

$$
M(a, b)=\max \{\widetilde{r}(a, b), \widetilde{r}(a, f a), \widetilde{r}(b, f b), \widetilde{r}(b, f a)\} .
$$

Then, $f$ has a fixed point. 
Proof. Starting with the given $a_{0}$, put $a_{n}=f^{n} a_{0}$. If $a_{n}=a_{n+1}$ for some $n \in \mathbb{N}$, then $a_{n}=f a_{n}$. Thus, $a_{n}$ is a fixed point of $f$. Therefore, we will assume that $a_{n} \neq a_{n+1}$ for all $n \in \mathbb{N}$. Since $a_{0} \preceq f a_{0}$ and $f$ is an increasing function, we obtain by induction that

$$
a_{0} \preceq f a_{0} \preceq f^{2} a_{0} \preceq \cdots \preceq f^{n} a_{0} \preceq f^{n+1} a_{0} \preceq \cdots .
$$

Step I: We will show that $\lim _{n \rightarrow \infty} \widetilde{r}\left(a_{n}, a_{n+1}\right)=0$. Since $a_{n} \preceq a_{n+1}$ for each $n \in \mathbb{N}$, then, by Inequality (1), we have

$$
\begin{aligned}
\psi\left(\widetilde{r}\left(a_{n}, a_{n+1}\right)\right) & =\psi\left(\widetilde{r}\left(f a_{n-1}, f a_{n}\right)\right) \leq \psi\left(\Omega\left(\widetilde{r}\left(f a_{n-1}, f a_{n}\right)\right)\right) \\
& \leq \psi\left(\Omega^{2}\left(\widetilde{r}\left(f a_{n-1}, f a_{n}\right)\right)\right) \leq \psi\left(M\left(a_{n-1}, a_{n}\right)\right)^{\beta\left(\widetilde{r}\left(a_{n-1}, a_{n}\right)\right)},
\end{aligned}
$$

where

$$
\begin{aligned}
M\left(a_{n-1}, a_{n}\right) & =\max \left\{\widetilde{r}\left(a_{n-1}, a_{n}\right), \widetilde{r}\left(a_{n-1}, a_{n}\right), \widetilde{r}\left(a_{n}, a_{n+1}\right), \widetilde{r}\left(a_{n}, a_{n}\right)\right\} \\
& \leq \max \left\{\widetilde{r}\left(a_{n-1}, a_{n}\right), \widetilde{r}\left(a_{n}, a_{n+1}\right)\right\} .
\end{aligned}
$$

If $\max \left\{\widetilde{r}\left(a_{n-1}, a_{n}\right), \widetilde{r}\left(a_{n}, a_{n+1}\right)\right\}=\widetilde{r}\left(a_{n}, a_{n+1}\right)$, then, from Inequality (2), we have

$$
\begin{aligned}
\psi\left(\widetilde{r}\left(a_{n}, a_{n+1}\right)\right) & \leq \psi\left(M\left(a_{n}, a_{n-1}\right)\right)^{\beta\left(\widetilde{r}\left(a_{n}, a_{n-1}\right)\right)} \\
& =\psi\left(\widetilde{r}\left(a_{n}, a_{n+1}\right)\right)^{\beta\left(\widetilde{r}\left(a_{n}, a_{n-1}\right)\right)} \\
& <\psi\left(\widetilde{r}\left(a_{n}, a_{n+1}\right)\right)^{\Omega^{-1}(1)} \leq \psi\left(\widetilde{r}\left(a_{n}, a_{n+1}\right)\right)
\end{aligned}
$$

which is a contradiction. Hence, $\max \left\{\widetilde{r}\left(a_{n-1}, a_{n}\right), \widetilde{r}\left(a_{n}, a_{n+1}\right)\right\}=\widetilde{r}\left(a_{n-1}, a_{n}\right)$, so, from Inequality (2),

$$
\psi\left(\widetilde{r}\left(a_{n}, a_{n+1}\right)\right) \leq \psi\left(M\left(a_{n-1}, a_{n}\right)\right)^{\beta\left(\widetilde{r}\left(a_{n}, a_{n-1}\right)\right)}=\psi\left(\widetilde{r}\left(a_{n-1}, a_{n}\right)\right)^{\beta\left(\widetilde{r}\left(a_{n}, a_{n-1}\right)\right)} .
$$

Therefore,

$$
\begin{aligned}
1 & \leq \psi\left(\widetilde{r}\left(a_{n}, a_{n+1}\right)\right) \\
& \leq \psi\left(\widetilde{r}\left(a_{n-1}, a_{n}\right)\right)^{\beta\left(\widetilde{r}\left(a_{n-1}, a_{n}\right)\right)} \\
& \leq \psi\left(\widetilde{r}\left(a_{n-2}, a_{n-1}\right)\right)^{\beta\left(\widetilde{r}\left(a_{n-1}, a_{n}\right)\right) \beta\left(\widetilde{r}\left(a_{n-2}, a_{n-1}\right)\right)} \\
& \vdots \\
& \leq \psi\left(\widetilde{r}\left(a_{0}, a_{1}\right)\right)^{\prod_{i=1}^{n} \beta\left(\widetilde{r}\left(a_{i-1}, a_{i}\right)\right)}
\end{aligned}
$$

Now, by definition of $\beta$, we have $\beta(t)<\Omega^{-1}(1) \leq 1, \forall t \in[0, \infty)$. Hence,

$$
\lim _{n \rightarrow \infty} \prod_{i=1}^{n} \beta\left(\widetilde{r}\left(a_{i}, a_{i-1}\right)\right)=0,
$$

and so

$$
\lim _{n \rightarrow \infty} \psi\left(\widetilde{r}\left(a_{1}, a_{0}\right)\right)^{\prod_{i=1}^{n} \beta\left(\widetilde{r}\left(a_{i}, a_{i-1}\right)\right)}=1 .
$$

Letting $n \rightarrow \infty$ in Inequality (3) and using Equation (4), we get

$$
\lim _{n \rightarrow \infty} \psi\left(\widetilde{r}\left(a_{n}, a_{n+1}\right)\right)=1,
$$

and since $\psi \in \Theta$, we obtain,

$$
\lim _{n \rightarrow \infty} \widetilde{r}\left(a_{n}, a_{n+1}\right)=0 .
$$


Step II: Now we will prove that $a_{n} \neq a_{m}$ for $n \neq m$. Suppose first that $a_{n}=a_{m}$ for some $n>m$, so we have $a_{n+1}=f a_{n}=f a_{m}=a_{m+1}$. By continuing this procedure, we conclude that $a_{n+k}=a_{m+k}$ for all $k \in \mathbb{N}$. Then, Inequality (1), Step I and being $\beta(t)<\Omega^{-1}(1) \leq 1, \forall t \in[0, \infty)$ imply that

$$
\begin{aligned}
\psi\left(\widetilde{r}\left(a_{m}, a_{m+1}\right)\right) & =\psi\left(\widetilde{r}\left(a_{n}, a_{n+1}\right)\right) \\
& \leq \psi\left(\Omega^{2}\left(\widetilde{r}\left(a_{n}, a_{n+1}\right)\right)\right) \\
& \leq \psi\left(M\left(a_{n-1}, a_{n}\right)\right)^{\beta\left(\widetilde{r}\left(a_{n-1}, a_{n}\right)\right)} \\
& \leq \psi\left(\max \left\{\widetilde{r}\left(a_{n-1}, a_{n}\right), \widetilde{r}\left(a_{n}, a_{n+1}\right)\right\}\right)^{\beta\left(\widetilde{r}\left(a_{n-1}, a_{n}\right)\right)} .
\end{aligned}
$$

If $\max \left\{\widetilde{r}\left(a_{n-1}, a_{n}\right), \widetilde{r}\left(a_{n}, a_{n+1}\right)\right\}=\widetilde{r}\left(a_{n}, a_{n+1}\right)$, then we have

$$
\psi\left(\widetilde{r}\left(a_{m}, a_{m+1}\right)\right) \leq \psi\left(\widetilde{r}\left(a_{n}, a_{n+1}\right)\right)^{\beta\left(\widetilde{r}\left(a_{n-1}, a_{n}\right)\right)}<\psi\left(\widetilde{r}\left(a_{n}, a_{n+1}\right)\right),
$$

a contradiction. If $\max \left\{\widetilde{r}\left(a_{n-1}, a_{n}\right), \widetilde{r}\left(a_{n}, a_{n+1}\right)\right\}=\widetilde{r}\left(a_{n-1}, a_{n}\right)$, then the above inequality becomes

$$
\begin{aligned}
& \psi\left(\widetilde{r}\left(a_{m}, a_{m+1}\right)\right) \leq \psi\left(\widetilde{r}\left(a_{n-1}, a_{n}\right)\right) \\
& \leq \psi\left(M\left(a_{n-2}, a_{n-1}\right)\right)^{\beta\left(\widetilde{r}\left(a_{n-2}, a_{n-1}\right)\right)} \\
& \leq \psi\left(\max \left\{\widetilde{r}\left(a_{n-2}, a_{n-1}\right), \widetilde{r}\left(a_{n-1}, a_{n}\right)\right\}\right)^{\beta\left(\widetilde{r}\left(a_{n-2}, a_{n-1}\right)\right)} \\
& <\psi\left(\max \left\{\widetilde{r}\left(a_{n-2}, a_{n-1}\right), \widetilde{r}\left(a_{n-1}, a_{n}\right)\right\}\right) \\
& \leq \psi\left(\widetilde{r}\left(a_{n-2}, a_{n-1}\right)\right) \quad(\text { from Inequalities (6) and (7)) } \\
& <\psi\left(\widetilde{r}\left(a_{m}, a_{m+1}\right)\right),
\end{aligned}
$$

a contradiction. Hence, from now on, we assume $a_{n} \neq a_{m}$ for all $n \neq m$.

Step III: Now, we will prove that $\left\{a_{n}\right\}$ is an $\widetilde{r}$-Cauchy sequence. Suppose to the contrary that there exists $\varepsilon>0$ for which we can find two subsequences $\left\{a_{m_{i}}\right\}$ and $\left\{a_{n_{i}}\right\}$ of $\left\{a_{n}\right\}$ such that $n_{i}$ is the smallest index where

$$
n_{i}>m_{i}>i \text { and } \widetilde{r}\left(a_{m_{i}}, a_{n_{i}}\right) \geq \varepsilon
$$

This means that

$$
\widetilde{r}\left(a_{m_{i}}, a_{n_{i}-2}\right), \widetilde{r}\left(a_{m_{i}}, a_{n_{i}-1}\right)<\varepsilon .
$$

From Inequality (8) and using the $\Omega$-rectangular inequality, we get

$$
\varepsilon \leq \widetilde{r}\left(a_{m_{i}}, a_{n_{i}}\right) \leq \Omega\left[\widetilde{r}\left(a_{m_{i}}, a_{m_{i}+1}\right)+\widetilde{r}\left(a_{m_{i}+1}, a_{n_{i}-1}\right)+\widetilde{r}\left(a_{n_{i}-1}, a_{n_{i}}\right)\right] .
$$

Taking the upper limit as $i \rightarrow \infty$, and using Equation (5), the above inequality turns into

$$
\Omega^{-1}(\varepsilon) \leq \limsup _{i \rightarrow \infty} \widetilde{r}\left(a_{m_{i}+1}, a_{n_{i}-1}\right) .
$$

Again, by using Inequality (8) and the $\Omega$-rectangular inequality, we obtain

$$
\widetilde{r}\left(a_{m_{i}+1}, a_{n_{i}-2}\right) \leq \Omega\left[\widetilde{r}\left(a_{m_{i}+1}, a_{m_{i}}\right)+\widetilde{r}\left(a_{m_{i}}, a_{n_{i}-1}\right)+\widetilde{r}\left(a_{n_{i}-1}, a_{n_{i}-2}\right)\right] .
$$

Taking the upper limit as $i \rightarrow \infty$, using Equation (5) and Inequality (9), we find that

$$
\limsup _{i \rightarrow \infty} \widetilde{r}\left(a_{m_{i}+1}, a_{n_{i}-2}\right) \leq \Omega(\varepsilon) .
$$


The rectangle inequality yields

$$
\widetilde{r}\left(a_{n_{i}-2}, a_{n_{i}+1}\right) \leq \Omega\left[\widetilde{r}\left(a_{n_{i}-2}, a_{n_{i}-1}\right)+\widetilde{r}\left(a_{n_{i}-1}, a_{n_{i}}\right)+\widetilde{r}\left(a_{n_{i}}, a_{n_{i}+1}\right)\right]
$$

and

$$
\widetilde{r}\left(a_{n_{i}-2}, a_{m_{i}+1}\right) \leq \Omega\left[\widetilde{r}\left(a_{n_{i}-2}, a_{n_{i}-1}\right)+\widetilde{r}\left(a_{n_{i}-1}, a_{m_{i}}\right)+\widetilde{r}\left(a_{m_{i}}, a_{m_{i}+1}\right)\right] .
$$

Using Inequalities (12) and (13), the definition of $M(a, b)$ gives that

$$
\begin{aligned}
& M\left(a_{m_{i}}, a_{n_{i}-2}\right) \\
& =\max \left\{\widetilde{r}\left(a_{m_{i}}, a_{n_{i}-2}\right), \widetilde{r}\left(a_{m_{i}}, a_{m_{i}+1}\right), \widetilde{r}\left(a_{n_{i}-2}, a_{n_{i}+1}\right), \widetilde{r}\left(a_{n_{i}-2}, a_{m_{i}+1}\right)\right\} \\
& \leq \max \left\{\begin{array}{c}
\widetilde{r}\left(a_{m_{i}}, a_{n_{i}-2}\right), \widetilde{r}\left(a_{m_{i}}, a_{m_{i}+1}\right), \\
\left.\Omega \widetilde{r}\left(a_{n_{i}-2}, a_{n_{i}-1}\right)+\widetilde{r}\left(a_{n_{i}-1}, a_{n_{i}}\right)+\widetilde{r}\left(a_{n_{i}}, a_{n_{i}+1}\right)\right], \\
\Omega\left[\widetilde{r}\left(a_{n_{i}-2}, a_{n_{i}-1}\right)+\widetilde{r}\left(a_{n_{i}-1}, a_{m_{i}}\right)+\widetilde{r}\left(a_{m_{i}}, a_{m_{i}+1}\right)\right]
\end{array}\right\} .
\end{aligned}
$$

Taking the upper limit as $i \rightarrow \infty$ and using Equation (5), Inequalities (9) and (11), we have

$$
\limsup _{i \rightarrow \infty} M\left(a_{m_{i}}, a_{n_{i}-2}\right) \leq \Omega(\varepsilon) \text {. }
$$

Now, from (1), we obtain

$$
\psi\left(\Omega^{2}\left[\widetilde{r}\left(a_{m_{i}+1}, a_{n_{i}-1}\right)\right]\right) \leq \psi\left(\Omega^{2}\left[\widetilde{r}\left(f a_{m_{i}}, f a_{n_{i}-2}\right)\right]\right) \leq \psi\left(M\left(a_{m_{i}}, a_{n_{i}-2}\right)\right)^{\beta\left(\widetilde{r}\left(a_{m_{i}}, a_{n_{i}-2}\right)\right.} .
$$

Again, taking the upper limit as $i \rightarrow \infty$ and combining Inequalities (10) and (11), the above inequality turns into

$$
\begin{aligned}
1<\psi(\Omega(\varepsilon)) & =\psi\left(\Omega^{2}\left[\Omega^{-1}[\varepsilon]\right]\right) \\
& \leq \psi\left(\Omega^{2}\left[\limsup _{i \rightarrow \infty} \widetilde{r}\left(a_{m_{i}+1}, a_{n_{i}-1}\right)\right]\right) \\
& \leq \psi\left(\left[\limsup _{i \rightarrow \infty} M\left(a_{m_{i}}, a_{n_{i}-2}\right)\right]\right)^{\beta\left(\widetilde{r}\left(a_{m_{i}}, a_{n_{i}-2}\right)\right)} \\
& <\psi(\Omega(\varepsilon))^{\Omega^{-1}[1]} \leq \psi(\Omega(\varepsilon))
\end{aligned}
$$

which is a contradiction. Consequently, $\left\{a_{n}\right\}$ is an $\widetilde{r}$-Cauchy sequence in $A$. Therefore, the sequence $\left\{a_{n}\right\} \widetilde{r}$-converges to some $c \in A$, that is, $\lim _{n \rightarrow \infty} \widetilde{r}\left(a_{n}, c\right)=0$.

Step IV: Now, we show that $c$ is a fixed point of $f$. Suppose that $f c \neq c$. Then, it follows that $a_{n}$ differs from both $f c$ and $c$ for $n$ sufficiently large and $a_{n} \preceq c$. Hence,

$$
\begin{aligned}
\psi\left(\widetilde{r}\left(a_{n+1}, f c\right)\right) & \leq \psi\left(\Omega^{2}\left[\widetilde{r}\left(a_{n+1}, f c\right)\right]\right) \leq \psi\left(M\left(a_{n}, c\right)\right)^{\beta\left(\widetilde{r}\left(a_{n}, c\right)\right)} \\
& =\psi\left(\left(\max \left\{\widetilde{r}\left(a_{n}, c\right), \widetilde{r}\left(a_{n}, f a_{n}\right), \widetilde{r}(c, f c), \widetilde{r}\left(c, f a_{n}\right)\right\}\right)\right)^{\beta\left(\widetilde{r}\left(a_{n}, c\right)\right)} .
\end{aligned}
$$

Thus,

$$
\begin{aligned}
& \psi(\widetilde{r}(c, f c)) \leq \liminf _{n \rightarrow \infty} \psi\left(\widetilde{r}\left(a_{n+1}, f c\right)\right) \leq \psi\left(\widetilde{r}\left(\liminf _{n \rightarrow \infty} a_{n+1}, f c\right)\right) \\
& \leq \psi\left(\limsup _{n \rightarrow \infty}\left(\max \left\{\widetilde{r}\left(a_{n}, c\right), \widetilde{r}\left(a_{n}, f a_{n}\right), \widetilde{r}(c, f c), \widetilde{r}\left(c, f a_{n}\right)\right\}\right)\right)^{\lim \sup _{n \rightarrow \infty} \beta\left(\widetilde{r}\left(a_{n}, c\right)\right)} \\
& \leq \psi(\widetilde{r}(c, f c))^{\lim \sup _{n \rightarrow \infty} \beta\left(\widetilde{r}\left(a_{n}, c\right)\right)} \\
& <\psi(\widetilde{r}(c, f c))^{\Omega^{-1}[1]} \leq \psi(\widetilde{r}(c, f c)),
\end{aligned}
$$

a contradiction. Thus, $c$ is a fixed point of $f$. 
The following examples illustrate the above obtained result.

Example 4. Let $A=\{\zeta, \lambda, \gamma, \omega, \eta\}$ be equipped with the order $\preceq$ given by

$$
\preceq=\{(\zeta, \zeta),(\lambda, \lambda),(\gamma, \gamma),(\omega, \omega),(\eta, \eta),(\omega, \zeta),(\omega, \lambda),(\omega, \gamma),(\omega, \eta),(\zeta, \gamma),(\lambda, \gamma),(\eta, \gamma)\}
$$

and let $d: A \times A \rightarrow[0,+\infty)$ be given as

$$
\begin{aligned}
& d(m, m)=0 \quad \text { for } m \in A ; \\
& d(m, n)=d(n, m) \quad \text { for } n, m \in A ; \\
& d(\zeta, \lambda)=0.45, \\
& d(\zeta, \gamma)=d(\zeta, \eta)=d(\lambda, \gamma)=d(\gamma, \eta)=0.05, \\
& d(\zeta, \omega)=d(\lambda, \omega)=d(\lambda, \eta)=d(\gamma, \omega)=d(\omega, \eta)=0.2,
\end{aligned}
$$

and $\widetilde{r}(m, n)=\sinh (d(m, n))$ for all $m, n \in A$. Then, one can easily check that $(A, \preceq, \widetilde{r})$ is a (complete) ordered $E R b M S$ with $\Omega(t)=\sinh \left(\frac{3}{2} t\right)$. Define $\psi:[0, \infty) \rightarrow[1, \infty)$ by $\psi(t)=\cosh (t)$. Consider the mapping $f: A \rightarrow A$ defined as

$$
f=\left(\begin{array}{lllll}
\zeta & \lambda & \gamma & \omega & \eta \\
\gamma & \gamma & \gamma & \zeta & \gamma
\end{array}\right)
$$

One easily can check that all the constraints of Theorem 2 are achieved with $\lambda(u)=\frac{1}{5} e^{-4 u} \leq 1.195 \approx$ $\sinh ^{-1}\left(\frac{3}{2}\right)$. The contractive Condition (1) is trivial except the case when $a \in\{\zeta, \lambda, \gamma, \eta\}, b=\omega$ (or vice versa) when it reduces to

$$
\begin{aligned}
\psi\left(\Omega^{2}(\widetilde{r}(f m, f n))\right) & =\cosh \left(\sinh \left(\frac{3}{2} \sinh \left(\frac{3}{2} \sinh (d(f m, f n))\right)\right)\right) \\
& \approx 1.006 \leq 1.017 \\
& \approx 1.020^{0.851} \approx \psi(M(m, n))^{\lambda(\widetilde{r}(m, n))},
\end{aligned}
$$

which implies that $f$ has a (unique) fixed point $\gamma$. Note that $(A, \widetilde{r})$ is neither a metric space, nor a rectangular metric space. For instance,

$$
\begin{aligned}
\widetilde{r}(\zeta, \lambda) & =\sinh (0.45) \approx 0.465>0.150 \\
& \approx \sinh (0.05)+\sinh (0.05)+\sinh (0.05) \\
& =\widetilde{r}(\zeta, \eta)+\widetilde{r}(\eta, \gamma)+\widetilde{r}(\gamma, \lambda) .
\end{aligned}
$$

Example 5. Let $A=\left\{0,1, \frac{1}{2}, \frac{1}{3}\right\}$ be equipped with the following partial order $\preceq$ :

$$
\preceq:=\left\{(0,0),(1,0),(1,1),\left(1, \frac{1}{2}\right),\left(\frac{1}{2}, \frac{1}{2}\right),\left(\frac{1}{3}, 1\right),\left(\frac{1}{3}, \frac{1}{2}\right),\left(\frac{1}{3}, \frac{1}{3}\right),\left(0, \frac{1}{3}\right)\right\} .
$$

Define the rectangular metric $d: A \times A \rightarrow \mathbb{R}^{+}$by

$$
d(a, b)= \begin{cases}0, & \text { if } a=b \\ \frac{1}{n^{2}}, & \text { if }\{a, b\}=\left\{0, \frac{1}{n}\right\} \\ 1, & \text { if } a \neq b, a, b \in A \backslash\{0\}\end{cases}
$$

and let $\widetilde{r}(a, b)=\sinh (d(a, b))$. It is easy to see that $(A, \widetilde{r})$ is a complete ERbMS. Define the self-map $f$ by

$$
f=\left(\begin{array}{cccc}
0 & 1 & \frac{1}{2} & \frac{1}{3} \\
0 & 0 & \frac{1}{3} & 0
\end{array}\right)
$$


We see that $f$ is an ordered increasing mapping. Define $\psi:[0, \infty) \rightarrow[1, \infty)$ by $\psi(t)=e^{\sqrt{t}}, \Omega(t)=\sinh (t)$ and $\beta(t)=\frac{1}{2+t} \leq 0.5 \leq 0.882 \approx \Omega^{-1}(1)$.

One can easily check that $f$ satisfies the Condition (1). We will present the nontrivial cases as follows:

1. $(a, b)=\left(\frac{1}{3}, 1\right)$. Then,

$$
\begin{aligned}
\psi\left(\Omega^{2}(\widetilde{r}(f a, f b))\right) & =e^{\sqrt{\sinh \left(\sinh \left(\sinh \left(d\left(f \frac{1}{3}, f 1\right)\right)\right)\right.}}=e^{\sqrt{\sinh (\sinh (\sinh (0)))}} \\
& =1 \leq 1.007 \approx 2.957^{0.315} \approx \psi(M(a, b))^{\beta(\widetilde{r}(a, b))} .
\end{aligned}
$$

2. $(a, b)=\left(\frac{1}{3}, \frac{1}{2}\right)$ or $(a, b)=\left(1, \frac{1}{2}\right)$. Then,

$$
\begin{aligned}
\psi\left(\Omega^{2}(\widetilde{r}(f a, f b))\right) & =e^{\sqrt{\sinh (\sinh (\sinh (d(f a, f b)))}}=e^{\sqrt{\sinh \left(\sinh \left(\sinh \left(\frac{1}{9}\right)\right)\right)}} \\
& =1.397 \leq 1.407 \approx 2.957^{0.315} \\
& =\psi(M(a, b))^{\beta(\widetilde{r}(a, b))} .
\end{aligned}
$$

Thus, all the conditions of Theorem 2 are satisfied and hence $f$ has a fixed point. Indeed, 0 is the fixed point of $f$.

By introducing the following concept (which is adapted from Definition 2.2 of [29]), we extend the result of Jleli and Samet [28]. Furthermore, we obtain some new generalizations of the Banach contraction principle.

Definition 6. Let $(A, \widetilde{r})$ be an ERbMS. The mapping $f: A \rightarrow A$ is said to be a $\psi_{\Omega}$-contraction, whenever there exists a function $\psi \in \Theta$ and functions $\beta_{1}, \beta_{2}, \beta_{3}, \beta_{4} \in \mathcal{F}_{\Omega}$ with $0 \leq \beta_{i}<\frac{\Omega^{-1}(1)}{4} ; i=1,2,3,4$ such that

$$
\begin{aligned}
& \psi\left(\Omega^{2}[\widetilde{r}(f a, f b)]\right) \\
& \leq \psi(\widetilde{r}(a, b))^{\beta_{1}(\widetilde{r}(a, b))} \psi(\widetilde{r}(a, f a))^{\beta_{2}(\widetilde{r}(a, f a))} \psi(\widetilde{r}(b, f b))^{\beta_{3}(\widetilde{r}(b, f b))} \psi(\widetilde{r}(b, f a))^{\beta_{4}(\widetilde{r}(b, f a))}
\end{aligned}
$$

for all $a, b \in A$.

Our second main result is the following:

Theorem 3. Let $(A, \widetilde{r})$ be a complete ERbMS and let $f: A \rightarrow A$ be a $\psi_{\Omega}$-contraction. Then, $f$ has a unique fixed point.

Proof. Let $a_{0} \in A$ be arbitrary. Define the sequence $\left\{a_{n}\right\}$ by $a_{n}=f a_{n-1}$. Assume that $a_{n} \neq a_{n+1}$ (If $a_{n_{0}}=a_{n_{0}+1}$ for some $n_{0} \in \mathbb{N}$, then $a_{n_{0}}$ is a fixed point of $f$ ), i.e., $\widetilde{r}\left(f a_{n-1}, f a_{n}\right)>0$ for all $n \in \mathbb{N} \cup\{0\}$.

First, we will prove that $\lim _{n \rightarrow \infty} \widetilde{r}\left(a_{n}, a_{n+1}\right)=0$. Since $f$ is a $\psi_{\Omega}$-contraction, using the Condition (14), we obtain that

$$
\begin{aligned}
& \psi\left(\widetilde{r}\left(a_{n}, a_{n+1}\right)\right)=\psi\left(\widetilde{r}\left(f a_{n-1}, f a_{n}\right)\right) \leq \psi\left(\Omega^{2}\left(\widetilde{r}\left(f a_{n-1}, f a_{n}\right)\right)\right) \\
& \leq \psi\left(\widetilde{r}\left(a_{n-1}, a_{n}\right)\right)^{\beta_{1}\left(\widetilde{r}\left(a_{n-1}, a_{n}\right)\right)} \psi\left(\widetilde{r}\left(a_{n-1}, f a_{n-1}\right)\right)^{\beta_{2}\left(\widetilde{r}\left(a_{n-1}, f a_{n-1}\right)\right)} \\
& \quad \psi\left(\widetilde{r}\left(a_{n}, f a_{n}\right)\right)^{\beta_{3}\left(\widetilde{r}\left(a_{n}, f a_{n}\right)\right)} \psi\left(\widetilde{r}\left(a_{n}, f a_{n-1}\right)\right)^{\beta_{4}\left(\widetilde{r}\left(a_{n}, f a_{n-1}\right)\right)} \\
& =\psi\left(\widetilde{r}\left(a_{n-1}, a_{n}\right)\right)^{\beta_{1}\left(\widetilde{r}\left(a_{n-1}, a_{n}\right)\right)} \psi\left(\widetilde{r}\left(a_{n-1}, a_{n}\right)\right)^{\beta_{2}\left(\widetilde{r}\left(a_{n-1}, a_{n}\right)\right)} \\
& \quad \psi\left(\widetilde{r}\left(a_{n}, a_{n+1}\right)\right)^{\beta_{3}\left(\widetilde{r}\left(a_{n}, a_{n+1}\right)\right)} \psi\left(\widetilde{r}\left(a_{n}, a_{n}\right)\right)^{\beta_{4}\left(\widetilde{r}\left(a_{n}, a_{n}\right)\right)} \\
& =\psi\left(\widetilde{r}\left(a_{n-1}, a_{n}\right)\right)^{\left[\beta_{1}\left(\widetilde{r}\left(a_{n-1}, a_{n}\right)\right)+\beta_{2}\left(\widetilde{r}\left(a_{n-1}, a_{n}\right)\right)\right]} \psi\left(\widetilde{r}\left(a_{n}, a_{n+1}\right)\right)^{\beta_{3}\left(\widetilde{r}\left(a_{n}, a_{n+1}\right)\right)} .
\end{aligned}
$$


Therefore,

$$
\psi\left(\widetilde{r}\left(a_{n}, a_{n+1}\right)\right)^{1-\beta_{3}\left(\widetilde{r}\left(a_{n}, a_{n+1}\right)\right)} \leq \psi\left(\widetilde{r}\left(a_{n-1}, a_{n}\right)\right)^{\beta_{1}\left(\widetilde{r}\left(a_{n-1}, a_{n}\right)\right)+\beta_{2}\left(\widetilde{r}\left(a_{n-1}, a_{n}\right)\right)},
$$

and so,

$$
\psi\left(\widetilde{r}\left(a_{n}, a_{n+1}\right)\right) \leq \psi\left(\widetilde{r}\left(a_{n-1}, a_{n}\right)\right)^{\frac{\beta_{1}\left(\widetilde{r}\left(a_{n-1}, a_{n}\right)\right)+\beta_{2}\left(\widetilde{r}\left(a_{n-1}, a_{n}\right)\right)}{1-\beta_{3}\left(\widetilde{r}\left(a_{n}, a_{n+1}\right)\right)}} .
$$

Let $\alpha_{n}=\frac{\beta_{1}\left(\widetilde{r}\left(a_{n-1}, a_{n}\right)\right)+\beta_{2}\left(\widetilde{r}\left(a_{n-1}, a_{n}\right)\right)}{1-\beta_{3}\left(\widetilde{r}\left(a_{n}, a_{n+1}\right)\right)}$. Then,

$$
\begin{aligned}
1 \leq \psi\left(\widetilde{r}\left(a_{n}, a_{n+1}\right)\right) & \leq \psi\left(\widetilde{r}\left(a_{n-1}, a_{n}\right)\right)^{\alpha_{n}} \\
& \leq \psi\left(\widetilde{r}\left(a_{n-2}, a_{n-1}\right)\right)^{\alpha_{n} \alpha_{n-1}} \\
& \vdots \\
& \leq \psi\left(\widetilde{r}\left(a_{0}, a_{1}\right)\right)^{\alpha_{n} \alpha_{n-1} \cdots \alpha_{1}} \\
& =\psi\left(\widetilde{r}\left(a_{0}, a_{1}\right)\right)^{\prod_{i=1}^{n} \alpha_{i}} .
\end{aligned}
$$

Note that, for $i=1,2, \ldots, n, \alpha_{i} \leq \frac{2}{3}<1$. Thus, $\lim _{n \rightarrow \infty} \prod_{i=1}^{n} \alpha_{i}=0$. Hence, $\lim _{n \rightarrow \infty} \psi\left(\widetilde{r}\left(a_{n}, a_{n+1}\right)\right)=1$. According to $\left(\psi_{2}\right)$, we conclude that

$$
\lim _{n \rightarrow \infty} \widetilde{r}\left(a_{n}, a_{n+1}\right)=0 .
$$

Now, we will prove that $\lim _{n \rightarrow \infty} \widetilde{r}\left(a_{n}, a_{n+2}\right)=0$. Since $f$ is a $\psi_{\Omega}$-contraction and using Condition (14), we obtain that

$$
\begin{aligned}
1 \leq & \psi\left(\widetilde{r}\left(a_{n}, a_{n+2}\right)\right) \leq \psi\left(\Omega\left(\widetilde{r}\left(a_{n}, a_{n+2}\right)\right)\right)=\psi\left(\Omega^{2}\left(\widetilde{r}\left(f a_{n-1}, f a_{n+1}\right)\right)\right) \\
\leq & \psi\left(\widetilde{r}\left(a_{n-1}, a_{n+1}\right)\right)^{\beta_{1}\left(\widetilde{r}\left(a_{n-1}, a_{n+1}\right)\right)} \psi\left(\widetilde{r}\left(a_{n-1}, f a_{n-1}\right)\right)^{\beta_{2}\left(\widetilde{r}\left(a_{n-1}, f a_{n-1}\right)\right)} \\
& \psi\left(\widetilde{r}\left(a_{n+1}, f a_{n+1}\right)\right)^{\beta_{3}\left(\widetilde{r}\left(a_{n+1}, f a_{n+1}\right)\right)} \psi\left(\widetilde{r}\left(a_{n+1}, f a_{n-1}\right)\right)^{\beta_{4}\left(\widetilde{r}\left(a_{n+1}, f a_{n-1}\right)\right)} \\
= & \psi\left(\widetilde{r}\left(a_{n-1}, a_{n+1}\right)\right)^{\beta_{1}\left(\widetilde{r}\left(a_{n-1}, a_{n+1}\right)\right)} \psi\left(\widetilde{r}\left(a_{n-1}, a_{n}\right)\right)^{\beta_{2}\left(\widetilde{r}\left(a_{n-1}, a_{n}\right)\right)} \\
& \psi\left(\widetilde{r}\left(a_{n+1}, a_{n+2}\right)\right)^{\beta_{3}\left(\widetilde{r}\left(a_{n+1}, a_{n+2}\right)\right)} \psi\left(\widetilde{r}\left(a_{n+1}, a_{n}\right)\right)^{\beta_{4}\left(\widetilde{r}\left(a_{n+1}, a_{n}\right)\right)} \\
\leq & \psi\left(\Omega\left[\widetilde{r}\left(a_{n-1}, a_{n+2}\right)+\widetilde{r}\left(a_{n+2}, a_{n}\right)+\widetilde{r}\left(a_{n}, a_{n+1}\right)\right]\right)^{\beta_{1}\left(\widetilde{r}\left(a_{n-1}, a_{n+1}\right)\right)} \\
& \psi\left(\widetilde{r}\left(a_{n-1}, a_{n}\right)\right)^{\beta_{2}\left(\widetilde{r}\left(a_{n-1}, a_{n}\right)\right)} \psi\left(\widetilde{r}\left(a_{n+1}, a_{n+2}\right)\right)^{\beta_{3}\left(\widetilde{r}\left(a_{n+1}, a_{n+2}\right)\right)} \psi\left(\widetilde{r}\left(a_{n+1}, a_{n}\right)\right)^{\beta_{4}\left(\widetilde{r}\left(a_{n+1}, a_{n}\right)\right)} \\
\leq & \psi\left(\Omega\left[\Omega\left[\widetilde{r}\left(a_{n-1}, a_{n}\right)+\widetilde{r}\left(a_{n}, a_{n+1}\right)+\widetilde{r}\left(a_{n+1}, a_{n+2}\right)\right]+\widetilde{r}\left(a_{n+2}, a_{n}\right)+\widetilde{r}\left(a_{n}, a_{n+1}\right)\right]\right)^{\beta_{1}\left(\widetilde{r}\left(a_{n-1}, a_{n+1}\right)\right)} \\
& \psi\left(\widetilde{r}\left(a_{n-1}, a_{n}\right)\right)^{\beta_{2}\left(\widetilde{r}\left(a_{n-1}, a_{n}\right)\right)} \psi\left(\widetilde{r}\left(a_{n+1}, a_{n+2}\right)\right)^{\beta_{3}\left(\widetilde{r}\left(a_{n+1}, a_{n+2}\right)\right)} \psi\left(\widetilde{r}\left(a_{n+1}, a_{n}\right)\right)^{\beta_{4}\left(\widetilde{r}\left(a_{n+1}, a_{n}\right)\right)} .
\end{aligned}
$$

Taking the upper limit as $n \rightarrow \infty$, we get

$$
\begin{aligned}
1 & \leq \limsup _{n \rightarrow \infty} \psi\left(\Omega\left(\widetilde{r}\left(a_{n}, a_{n+2}\right)\right)\right) \\
& \leq \psi\left[\Omega\left(\Omega(0)+\limsup _{n \rightarrow \infty} \widetilde{r}\left(a_{n+2}, a_{n}\right)\right)\right]^{\frac{\Omega^{-1}(1)}{4}} \\
& =\psi\left[\Omega\left(\limsup _{n \rightarrow \infty} \widetilde{r}\left(a_{n+2}, a_{n}\right)\right)\right]^{\frac{\Omega^{-1}(1)}{4}} .
\end{aligned}
$$

Since $\frac{\Omega^{-1}(1)}{4}<\frac{1}{4}$, the above can only happen if

$$
\psi\left(\Omega\left(\limsup _{n \rightarrow \infty} \widetilde{r}\left(a_{n+2}, a_{n}\right)\right)\right)=1 .
$$


Thus, it must hold

$$
\Omega\left(\limsup _{n \rightarrow \infty} \widetilde{r}\left(a_{n+2}, a_{n}\right)\right)=0 .
$$

However,

$$
0 \leq \limsup _{n \rightarrow \infty} \widetilde{r}\left(a_{n+2}, a_{n}\right) \leq \Omega\left(\limsup _{n \rightarrow \infty} \widetilde{r}\left(a_{n+2}, a_{n}\right)\right)=0 .
$$

Hence,

$$
\limsup _{n \rightarrow \infty} \widetilde{r}\left(a_{n}, a_{n+2}\right)=0 .
$$

In order to show that $\left\{a_{n}\right\}$ is an $\widetilde{r}$-Cauchy sequence, suppose the contrary. Then, there exists $\varepsilon>0$ for which we can find two subsequences $m_{i}$ and $n_{i}$ such that $n_{i}$ is the smallest index where

$$
n_{i}>m_{i}>i \text { and } \widetilde{r}\left(a_{m_{i}}, a_{n_{i}}\right) \geq \varepsilon .
$$

This means that

$$
\widetilde{r}\left(a_{m_{i}}, a_{n_{i}-1}\right)<\varepsilon \text { and } \widetilde{r}\left(a_{m_{i}}, a_{n_{i}-2}\right)<\varepsilon .
$$

Rectangle inequality implies

$$
\widetilde{r}\left(a_{m_{i}-1}, a_{n_{i}-2}\right) \leq \Omega\left[\widetilde{r}\left(a_{m_{i}-1}, a_{m_{i}}\right)+\widetilde{r}\left(a_{m_{i}}, a_{n_{i}-1}\right)+\widetilde{r}\left(a_{n_{i}-1}, a_{n_{i}-2}\right)\right] .
$$

Taking the upper limit as $i \rightarrow \infty$, and using Equation (15) and Inequality (18), we get

$$
\limsup _{i \rightarrow \infty} \widetilde{r}\left(a_{m_{i}-1}, a_{n_{i}-2}\right) \leq \Omega(\varepsilon) .
$$

From Inequality (18), we have

$$
\widetilde{r}\left(a_{m_{i}}, a_{n_{i}-1}\right)<\varepsilon \leq \Omega(\varepsilon),
$$

which yields that

$$
\limsup _{n \rightarrow \infty} \widetilde{r}\left(a_{m_{i}}, a_{n_{i}-1}\right) . \leq \Omega(\varepsilon) .
$$

Combining rectangle inequalities and Inequality (17), we get

$$
\begin{aligned}
\varepsilon & \leq \Omega\left(\widetilde{r}\left(a_{m_{i}}, a_{n_{i}}\right)\right) \\
& \leq \Omega\left(\widetilde{r}\left(a_{m_{i}}, a_{n_{i}-1}\right)+\widetilde{r}\left(a_{n_{i}-1}, a_{n_{i}-2}\right)+\widetilde{r}\left(a_{n_{i}-2}, a_{n_{i}}\right)\right) .
\end{aligned}
$$

Taking the upper limit as $i \rightarrow \infty$ and using Equation (16), Inequalities (20) and (18), we get

$$
\limsup _{i \rightarrow \infty} \widetilde{r}\left(a_{m_{i}}, a_{n_{i}-1}\right) \geq \Omega^{-1}(\varepsilon) .
$$

On the other hand, we have

$$
\begin{aligned}
\psi( & \left(\widetilde{r}\left(a_{m_{i}}, a_{n_{i-1}}\right)\right) \\
= & \psi\left(\widetilde{r}\left(f a_{m_{i}-1}, f a_{n_{i}-2}\right)\right) \\
\leq & \psi\left(\Omega^{2}\left(\widetilde{r}\left(f a_{m_{i}-1}, f a_{n_{i}-2}\right)\right)\right) \\
\leq & \psi\left(\widetilde{r}\left(a_{m_{i}-1}, a_{n_{i}-2}\right)\right)^{\beta_{1}\left(\widetilde{r}\left(a_{m_{i}-1}, a_{n_{i}-2}\right)\right)} \psi\left(\widetilde{r}\left(a_{m_{i}-1}, f a_{m_{i}-1}\right)\right)^{\beta_{2}\left(\widetilde{r}\left(a_{m_{i}-1}, f a_{m_{i}-1}\right)\right)} \\
& \psi\left(\widetilde{r}\left(a_{n_{i}-2}, f a_{n_{i}-2}\right)\right)^{\beta_{3}\left(\widetilde{r}\left(a_{n_{i}-2}, f a_{n_{i}-2}\right)\right)} \psi\left(\widetilde{r}\left(a_{n_{i}-2}, f a_{m_{i}-1}\right)\right)^{\beta_{4}\left(\widetilde{r}\left(a_{n_{i}-2}, f a_{m_{i}-1}\right)\right)} \\
= & \psi\left(\widetilde{r}\left(a_{m_{i}-1}, a_{n_{i}-2}\right)\right)^{\beta_{1}\left(\widetilde{r}\left(a_{m_{i}-1}, a_{n_{i}-2}\right)\right)} \psi\left(\widetilde{r}\left(a_{m_{i}-1}, a_{m_{i}}\right)\right)^{\beta_{2}\left(\widetilde{r}\left(a_{m_{i}-1}, a_{m_{i}}\right)\right)} \\
& \psi\left(\widetilde{r}\left(a_{n_{i}-2}, a_{n_{i}-1}\right)\right)^{\beta_{3}\left(\widetilde{r}\left(a_{n_{i}-2}, a_{n_{i}-1}\right)\right)} \psi\left(\widetilde{r}\left(a_{n_{i}-2}, a_{m_{i}}\right)\right)^{\beta_{4}\left(\widetilde{r}\left(a_{n_{i}-2}, a_{m_{i}}\right)\right)} .
\end{aligned}
$$


Now, taking the upper limit as $i \rightarrow \infty$ in the above inequality and using $\left(\psi_{1}\right)$ and Inequality (22), we have

$$
\begin{aligned}
\psi(\Omega[\varepsilon])= & \psi\left(\Omega^{2}\left[\Omega^{-1}(\varepsilon)\right]\right) \\
\leq & \psi\left(\Omega^{2}\left[\limsup _{i \rightarrow \infty} \widetilde{r}\left(a_{m_{i}}, a_{n_{i}-1}\right)\right]\right) \\
\leq & \psi\left(\limsup _{i \rightarrow \infty} \widetilde{r}\left(a_{m_{i}-1}, a_{n_{i}-2}\right)\right) \limsup _{i \rightarrow \infty} \beta_{1}\left(\widetilde{r}\left(a_{m_{i}-1}, a_{n_{i}-2}\right)\right) \\
& \psi\left(\limsup _{i \rightarrow \infty} \widetilde{r}\left(a_{m_{i}-1}, a_{m_{i}}\right)\right) \\
& \psi\left(\limsup _{i \rightarrow \infty} \widetilde{r}\left(a_{n_{i}-2}, a_{n_{i}-1}\right)\right)^{\left.\operatorname{lims} \beta_{2}\left(a_{m_{i}-1}, a_{m_{i}}\right)\right)} \\
& \psi\left(\limsup _{i \rightarrow \infty} \widetilde{r}\left(a_{n_{i}-2}, a_{m_{i}}\right)\right)^{\left.\lim \left(a_{n_{i}-2}, a_{n_{i}-1}\right)\right)}
\end{aligned}
$$

which further implies, from Equation (15), Inequalities (18) and (19), that

$$
1<\psi(\Omega[\varepsilon]) \leq \psi(\Omega[\varepsilon])^{\frac{\Omega^{-1}(1)}{2}}<\psi(\Omega[\varepsilon]),
$$

which is a contradiction. Thus, we have proved that $\left\{a_{n}\right\}$ is an $\widetilde{r}$-Cauchy sequence. The completeness of $A$ ensures that there exists $a^{*} \in A$ such that, $a_{n} \rightarrow a^{*}$ as $n \rightarrow \infty$. Suppose that $a^{*} \neq f a^{*}$; then, from Condition (14), we have

$$
\begin{aligned}
& \psi\left(\widetilde{r}\left(a_{n}, f a^{*}\right)\right) \\
& =\psi\left(\widetilde{r}\left(f a_{n-1}, f a^{*}\right)\right) \\
& \leq \psi\left(\widetilde{r}\left(a_{n-1}, a^{*}\right)\right)^{\beta_{1}\left(\widetilde{r}\left(a_{n-1}, a^{*}\right)\right)} \psi\left(\widetilde{r}\left(a_{n-1}, f a_{n-1}\right)\right)^{\beta_{2}\left(\widetilde{r}\left(a_{n-1}, f a_{n-1}\right)\right)} \\
& \quad \psi\left(\widetilde{r}\left(a^{*}, f a^{*}\right)\right)^{\beta_{3}\left(\widetilde{r}\left(a^{*}, f a^{*}\right)\right)} \psi\left(\widetilde{r}\left(a^{*}, f a_{n-1}\right)\right)^{\beta_{4}\left(\widetilde{r}\left(a^{*}, f a_{n-1}\right)\right)} \\
& \leq \psi\left(\widetilde{r}\left(a_{n-1}, a^{*}\right)\right)^{\beta_{1}\left(\widetilde{r}\left(a_{n-1}, a^{*}\right)\right)} \psi\left(\widetilde{r}\left(a_{n-1}, a_{n}\right)\right)^{\beta_{2}\left(\widetilde{r}\left(a_{n-1}, a_{n}\right)\right)} \\
& \quad \psi\left(\widetilde{r}\left(a^{*}, f a^{*}\right)\right)^{\beta_{3}\left(\widetilde{r}\left(a^{*}, f a^{*}\right)\right)} \psi\left(\widetilde{r}\left(a^{*}, a_{n}\right)\right)^{\beta_{4}\left(\widetilde{r}\left(a^{*}, a_{n}\right)\right)} .
\end{aligned}
$$

Taking the upper limit as $n \rightarrow \infty$ in the above inequality and using $\left(\psi_{1}\right)$ and Equation (15), we have

$$
\begin{aligned}
& 1< \psi\left(\widetilde{r}\left(a^{*}, f a^{*}\right)\right) \leq \psi\left(\widetilde{r}\left(\liminf _{n \rightarrow \infty} a_{n}, f a^{*}\right)\right) \leq \liminf _{n \rightarrow \infty} \psi\left(\widetilde{r}\left(a_{n}, f a^{*}\right)\right) \\
& \leq \limsup _{n \rightarrow \infty} \psi\left(\widetilde{r}\left(a_{n}, f a^{*}\right)\right) \\
& \leq \limsup _{n \rightarrow \infty} \psi\left(\widetilde{r}\left(a_{n-1}, a^{*}\right)\right) \limsup _{n \rightarrow \infty} \beta_{1}\left(\widetilde{r}\left(a_{n-1}, a^{*}\right)\right) \\
& \quad \limsup _{n \rightarrow \infty} \psi\left(\widetilde{r}\left(a_{n-1}, a_{n}\right)\right) \limsup _{n \rightarrow \infty} \beta_{2}\left(\widetilde{r}\left(a_{n-1}, a_{n}\right)\right) \\
& \limsup _{n \rightarrow \infty} \psi\left(\widetilde{r}\left(a^{*}, f a^{*}\right)\right)^{\limsup \sup _{n \rightarrow \infty} \beta_{3}\left(\widetilde{r}\left(a^{*}, f a^{*}\right)\right)} \\
& \limsup _{n \rightarrow \infty} \psi\left(\widetilde{r}\left(a^{*}, a_{n}\right)\right)^{\lim \sup _{n \rightarrow \infty} \beta_{4}\left(\widetilde{r}\left(a^{*}, a_{n}\right)\right)} \\
& \leq \psi\left(\widetilde{r}\left(a^{*}, f a^{*}\right)\right)^{\beta_{3}\left(\widetilde{r}\left(a^{*}, f a^{*}\right)\right)} \leq \psi\left(\widetilde{r}\left(a^{*}, f a^{*}\right)\right)^{\frac{\Omega^{-1}}{4}}<\psi\left(\widetilde{r}\left(a^{*}, f a^{*}\right)\right),
\end{aligned}
$$

which is a contradiction, that is, $a^{*}=f a^{*}$. Thus, $f$ has a fixed point. The uniqueness is straightforward. 
Taking various functions $\psi \in \Theta$ (see Remark 1 ), several corollaries of the previous theorem are obtained. For instance, taking $\psi(t)=e^{t}, \psi(t)=e^{t e^{t}}, \psi(t)=\frac{2 e^{t e^{t}}}{1+e^{t e^{t}}}$, or $\psi(t)=1+\ln (1+t)$, we get

Corollary 1. Let $(A, \widetilde{r})$ be a complete ERbMS and $f: A \rightarrow A$ be a mapping such that one of the following conditions is satisfied for all $a, b \in A$ :

(1)

$$
\begin{aligned}
\widetilde{r}(f a, f b) \leq \beta_{1}(\widetilde{r}(a, b)) \widetilde{r}(a, b)+\beta_{2}(\widetilde{r}(a, f a)) \widetilde{r}(a, f a) \\
+\beta_{3}(\widetilde{r}(b, f b)) \widetilde{r}(b, f b)+\beta_{4}(\widetilde{r}(b, f a)) \widetilde{r}(b, f a) ;
\end{aligned}
$$

(2)

$$
\begin{aligned}
\widetilde{r}(f a, f b) e^{\widetilde{r}(f a, f b)} \leq & \beta_{1}(\widetilde{r}(a, b)) \widetilde{r}(a, b) e^{\widetilde{r}(a, b)}+\beta_{2}(\widetilde{r}(a, f a)) \widetilde{r}(a, f a) e^{\widetilde{r}(a, f a)} \\
& +\beta_{3}(\widetilde{r}(b, f b)) \widetilde{r}(b, f b) e^{\widetilde{r}(b, f b)}+\beta_{4}(\widetilde{r}(b, f a)) \widetilde{r}(b, f a) e^{\widetilde{r}(b, f a)} ;
\end{aligned}
$$

(3)

$$
\begin{aligned}
& 1+\ln (1+\widetilde{r}(f a, f b)) \\
& \leq[1+\ln (1+\widetilde{r}(a, b))]^{\beta_{1}(\widetilde{r}(a, b))}[1+\ln (1+\widetilde{r}(a, f a))]^{\beta_{2}(\widetilde{r}(a, f a))} \\
& \quad[1+\ln (1+\widetilde{r}(b, f b))]^{\beta_{3}(\widetilde{r}(b, f b))}[1+\ln (1+\widetilde{r}(b, f a))]^{\beta_{4}(\widetilde{r}(b, f a)),}
\end{aligned}
$$

where $\beta_{i}, \beta_{2}, \beta_{3}, \beta_{4} \in \mathcal{F}_{\Omega}$ are such that $0 \leq \beta_{i}(t)<\frac{\Omega^{-1}(1)}{4}$ for all $t>0$ and $i=1,2,3,4$. Then, $f$ has a unique fixed point.

Taking $\beta_{1}=\beta_{4}=0$ and $\beta_{2}=\beta_{3}=k$ in (1) of the above result, we obtain Theorem 2.7 of [29] in the framework of non-ordered ERbMS.

Example 6. Let $A=\{0\} \cup\left\{\frac{1}{n}: n \in \mathbb{N}\right\}$ be endowed with the rectangular metric

$$
d(a, b)= \begin{cases}0, & \text { if } a=b, \\ \frac{1}{n}, & \text { if }\{a, b\}=\left\{0, \frac{1}{n}\right\}, \\ 1, & \text { otherwise, }\end{cases}
$$

for all $a, b \in A$ and $\widetilde{r}(a, b)=e^{d(a, b)}-1$. Define $f: A \rightarrow A$ and $\psi: R^{+} \rightarrow[1, \infty)$ by $f a=\frac{1}{8} a$ and $\psi(t)=1+\ln (1+t), \beta_{i}(t)=\frac{2}{7+\ln (1+t)}$ for $i=1,2,3,4$. Let $\{a, b\}=\left\{0, \frac{1}{n}\right\}$. Then, $d(f a, f b)=\frac{1}{8 n}$. Therefore, $1+\ln (1+\widetilde{r}(f a, f b))=1+\frac{1}{8 n}$, and

$$
\begin{aligned}
{[1+\ln (1+\widetilde{r}(a, b))]^{\beta_{1}(\widetilde{r}(a, b))}[1+\ln (1+\widetilde{r}(a, f a))]^{\beta_{2}(\widetilde{r}(a, f a))} } & {[1+\ln (1+\widetilde{r}(b, f b))]^{\beta_{3}(\widetilde{r}(b, f b))}[1+\ln (1+\widetilde{r}(b, f a))]^{\beta_{4}(\widetilde{r}(b, f a))} } \\
= & {\left[1+\frac{1}{n}\right]^{\beta_{1}\left(e^{\frac{1}{n}}-1\right)}[1]^{\beta_{2}(0)}[2]^{\beta_{3}(e-1)}\left[1+\frac{1}{n}\right]^{\beta_{4}\left(e^{\frac{1}{n}}-1\right)} } \\
= & {\left[1+\frac{1}{n}\right]^{\frac{2}{n}+7}[2]^{\frac{2}{8}} . }
\end{aligned}
$$

Since $[2]^{\frac{2}{8}}>1$ and $\left[1+\frac{1}{8 n}\right] \leq\left[1+\frac{1}{n}\right]^{\frac{2}{n}+7}$, we get

$$
\left[1+\frac{1}{8 n}\right] \leq\left[1+\frac{1}{n}\right]^{\frac{2}{n+7}}[2]^{\frac{2}{8}} .
$$


Let $a, b \in\left\{\frac{1}{n}: n \in \mathbb{N}\right\}$. Then, $1+\ln (1+\widetilde{r}(f a, f b))=[1+1]=2$, and

$$
\begin{aligned}
{[1+} & \ln (1+\widetilde{r}(a, b))]^{\beta_{1}(\widetilde{r}(a, b))}[1+\ln (1+\widetilde{r}(a, f a))]^{\beta_{2}(\widetilde{r}(a, f a))} \\
& {[1+\ln (1+\widetilde{r}(b, f b))]^{\beta_{3}(\widetilde{r}(b, f b))}[1+\ln (1+\widetilde{r}(b, f a))]^{\beta_{4}(\widetilde{r}(b, f a))} } \\
= & {[1+1]^{\beta_{1}(e-1)}[1+1]^{\beta_{2}(e-1)}[1+1]^{\beta_{3}(e-1)}[1+1]^{\beta_{4}(e-1)} } \\
= & {[2]^{\frac{2}{8}}[2]^{\frac{2}{8}}[2]^{\frac{2}{8}}[2]^{\frac{2}{8}} . }
\end{aligned}
$$

Thus, $2 \leq[2]^{4\left(\frac{1}{4}\right)}$. Hence, $f$ is a $\psi_{\Omega^{-c o n t r a c t i o n}}$ and so all conditions of Corollary 1 are satisfied and $f$ has a fixed point.

\section{Some Consequences in Rectangular $b$-Metric Spaces}

Corollary 2. Let $\left(A, r_{b}\right)$ be a rectangular b-metric space and $f: A \rightarrow A$ be such that

$$
\begin{aligned}
& \psi\left(\sinh \left(s \cdot \sinh \left(s \cdot\left[\sinh \left(r_{b}(f a, f b)\right)\right]\right)\right)\right) \\
& \leq \psi\left(\sinh \left(r_{b}(a, b)\right)\right)^{\beta_{1}\left(\sinh \left(r_{b}(a, b)\right)\right)} \psi\left(\sinh \left(r_{b}(a, f a)\right)\right)^{\beta_{2}\left(\sinh \left(r_{b}(a, f a)\right)\right)} \\
& \quad \psi\left(\sinh \left(r_{b}(b, f b)\right)\right)^{\beta_{3}\left(\sinh \left(r_{b}(b, f b)\right)\right)} \psi\left(\sinh \left(r_{b}(b, f a)\right)\right)^{\beta_{4}\left(\sinh \left(r_{b}(b, f a)\right)\right)}
\end{aligned}
$$

for all $a, b \in A$, where $\psi \in \Theta$ and $\beta_{1}, \beta_{2}, \beta_{3}, \beta_{4}$ are positive real functions such that $0 \leq \beta_{i}(t)<\frac{\sinh ^{-1}(1)}{4}$ for all $t \geq 0$ and $i=1,2,3,4$. Then, $f$ has a unique fixed point.

Corollary 3. Let $\left(A, r_{b}\right)$ be a rectangular $b$-metric space. Let $f: A \rightarrow A$ be such that

$$
\begin{aligned}
& \psi\left(e^{s \cdot e^{s \cdot e^{r} b(f a, f b)}-1}-1-1\right) \\
& \leq \psi\left(e^{r_{b}(a, b)}-1\right)^{\beta_{1}\left(e^{r_{b}(a, b)}-1\right)} \psi\left(e^{r_{b}(a, f a)}-1\right)^{\beta_{2}\left(e^{r_{b}(a, f a)}-1\right)} \\
& \quad \psi\left(e^{r_{b}(b, f b)}-1\right)^{\beta_{3}\left(e^{r_{b}(b, f b)}-1\right)} \psi\left(e^{r_{b}(b, f a)}-1\right)^{\beta_{4}\left(e^{r_{b}(b, f a)}-1\right)}
\end{aligned}
$$

for all $a, b \in A$ where $\psi \in \Theta$ and $\beta_{1}, \beta_{2}, \beta_{3}, \beta_{4}$ are positive real functions such that $0 \leq \beta_{i}(t)<\frac{\ln (2)}{4}$ for all $t \geq 0$ and $i=1,2,3,4$. Then, $f$ has a unique fixed point.

\section{Conclusions}

We have introduced the class of extended rectangular $b$-metric spaces (ERbMS) as a generalization of both rectangular metric and rectangular $b$-metric spaces, and we proved some fixed point results on complete ordered ERbMS with certain contractions. Moreover, one can easily see that the set of fixed points of a mapping is well ordered if and only if the mapping has a unique fixed point. Furthermore, by introducing the concept of $\psi_{\Omega}$-contraction, we extend the result of Jleli and Samet and we obtained some new generalizations of the Banach contraction principle. It would be very interesting to analyze the existing literature in light of the new defined metric (ERbMS).

Author Contributions: Conceptualization, Z.M., V.P., M.M.M.J. and Z.K.; methodology, Z.M., V.P., M.M.M.J. and Z.K.; investigation, Z.M., V.P., M.M.M.J. and Z.K.; writing-original draft preparation, V.P. and Z.K.; writing-review and editing, Z.M. and M.M.M.J.

Funding: The publication of this article was funded by the Qatar National Library.

Acknowledgments: The publication of this article was funded by the Qatar National Library. The authors are highly appreciated the referees efforts of this paper who helped us to improve it in several places.

Conflicts of Interest: The authors declare no conflict of interest. 


\section{References}

1. Border, K. Fixed Point Theorems with Applications to Economics and Game Theory; Cambridge University Press: Cambridge, UK, 1985.

2. Kakutani, S. A generalization of Brouwer's fixed point theorem. Duke Math. J. 1941, 8, 457-459. [CrossRef]

3. Branciari, A. A fixed point theorem of Banach-Caccioppoli type on a class of generalized metric spaces. Publ. Math. Debr. 2000, 57, 31-37.

4. George, R.; Redenovic, S.; Reshma, K.P.; Shukla, S. Rectangular b-metric space and contraction principles. J. Nonlinear Sci. Appl. 2015, 8, 1005-1013. [CrossRef]

5. Czerwik, S. Contraction mappings in b-metric spaces. Acta Math. Inf. Univ. Ostrav. 1993, 1, 5-11.

6. Aydi, H.; Bota, M.; Karapinar, E.; Moradi, S. A Common Fixed Point For Weak-Phi-Contractions on b-Metric Spaces. Fixed Point Theory 2012, 13, 337-346.

7. Czerwik, S. Nonlinear set-valued contraction mappings in b-metric spaces. Atti Sem. Mat. Fis. Univ. Modena 1998, 46, 263-276.

8. Karapinar, E.; Piri, H.; AlSulami, H.H. Fixed Points of Generalized F-Suzuki Type Contraction in Complete b-Metric Spaces. Discret. Dyn. Nat. Soc. 2015, 2015, 969726.

9. Mustafa, Z.; Jaradat, M.M.M.; Jaradat, H.M. Some common fixed point results of graphs on $b$-metric space. J. Nonlinear Sci. Appl. 2016, 9, 4838-4851. [CrossRef]

10. Sintunavarat, W.; Plubtieng, S.; Katchang, P. Fixed point result and applications on $b$-metric space endowed with an arbitrary binary relation. Fixed Point Theory Appl. 2013, 2013, 296. [CrossRef]

11. Jaradat, M.M.M.; Mustafa, Z.; Arshad, M.; Khan, S.U.; Ahmad, J. Some fixed point results on G-metric and $G_{b}$-metric spaces. Demonstr. Math. 2017, 50, 190-207.

12. Mustafa, Z.; Roshan, J.R.; Parvaneh, V. Existence Of Tripled Coincidence Point In Ordered $G_{b}$-Metric Spaces and Applications To System Of Integral Equations. J. Inequal. Appl. 2013, 2013, 453. [CrossRef]

13. Mustafa, Z.; Roshan, J.R.; Parvaneh, V.; Kadelburg, Z. Some common fixed point results in ordered partial b-metric space. J. Inequal. Appl. 2013, 2013, 562. [CrossRef]

14. Mustafa, Z.; Roshan, J.R.; Parvaneh, V.; Kadelburg, Z. Fixed point theorems for weakly T-Chatterjea and weakly T-Kannan contractions in b-metric spaces. J. Inequal. Appl. 2014, 2014, 46. [CrossRef]

15. Mustafa, Z.; Jaradat, M.M.M.; Ansari, A.; Popović, B.Z.; Jaradat, H. C-class functions with new approach to coincidence point results for generalized $(\psi, \varphi)$-weakly contractions in ordered b-metric spaces. SpringerPlus 2016, 5, 802. [CrossRef] [PubMed]

16. Mustafa, Z.; Parvaneh, V.; Roshan, J.R.; Kadelburg, Z. b2-Metric Spaces and Some Fixed Point Theorems. Fixed Point Theory Appl. 2014, 2014, 144. [CrossRef]

17. Ansari, A.H.; Chandok, S.; Ionescu, C. Fixed point theorems on b-metric spaces for weak contractions with auxiliary functions. J. Inequal. Appl. 2014, 2014, 429. [CrossRef]

18. Zabihi, F.; Razani, A. Fixed Point Theorems for Hybrid Rational Geraghty Contractive Mappings in Ordered b-Metric Spaces. J. Appl. Math. 2014, 2014, 929821. [CrossRef]

19. Parvaneh, V. Fixed points of $(\psi, \varphi)_{\Omega}$-contractive mappings in ordered $p$-metric spaces. submitted.

20. Kadelburg, Z.; Radenović, S. Fixed point results in generalized metric spaces without Hausdorff property. Math. Sci. 2014, 8, 125. [CrossRef]

21. Kadelburg, Z.; Radenović, S. On generalized metric spaces: a survey. TWMS J. Pure Appl. Math. 2014, 5, 3-13.

22. Karapinar, E.; Aydi, H.; Samet, B. Fixed points for generalized (alpha,psi)-contractions on generalized metric spaces. J. Inequal. Appl. 2014, 2014, 229.

23. Kirk, W.; Shahzad, N. Fixed Point Theory in Distance Spaces; Springer: Cham, Switzerland, 2014; Volume XI, p. 173.

24. Suzuki, T. Generalized metric spaces do not have the compatible topology. Abstr. Appl. Anal. 2014, 2014, 458098. [CrossRef]

25. Roshan, J.R.; Parvaneh, V.; Kadelburg, Z.; Hussain, N. New fixed point results in b-rectangular metric spaces. Nonlinear Anal. Model. Control 2016, 21, 614-634. [CrossRef]

26. Samet, B. Discussion on 'A fixed point theorem of Banach-Caccioppoli type on a class of generalized metric spaces' by A. Branciari. Publ. Math. Debr. 2010, 76, 493-494.

27. Sarma, I.R.; Rao, J.M.; Rao, S.S. Contractions over generalized metric spaces. J. Nonlinear Sci. Appl. 2009, 2, 180-182. [CrossRef] 
28. Jleli, M.; Samet, B. A new generalization of the Banach contraction principle. J. Inequal. Appl. 2014, $2014,38$. [CrossRef]

29. Hussain, N.; Parvaneh, V.; Samet, B.; Vetro, C. Some fixed point theorems for generalized contractive mappings in complete metric spaces. Fixed Point Theory Appl. 2015, 2015, 185. [CrossRef]

30. Jiang, S.; Li, Z.; Damjanović, B. A note on "Some fixed point theorems for generalized contractive mappings in complete metric spaces". Fixed Point Theory Appl. 2016, 2016, 62. [CrossRef]

(C) 2019 by the authors. Licensee MDPI, Basel, Switzerland. This article is an open access article distributed under the terms and conditions of the Creative Commons Attribution (CC BY) license (http:/ / creativecommons.org/licenses/by/4.0/). 